\title{
Starting a Revolution: A New Approach to Scanning Beam Microscopy
}

\author{
L.G. Lechner* \\ * Material Science Group, Center for Electron Microscopy, Ulm University, Ulm, Germany
}

The operating principle of a scanning beam microscope (SBM) is very similar to that of cathode ray tube (CRT) television [1]. This is no surprise since the background of Max Knoll, the inventor of the scanning electron microscope (SEM), was in television engineering. Arguably, during the past 70 years we have seen dramatic improvement in SEM performance and capabilities. Nevertheless, the basic operating principle has remained unchanged. Even though the CRT has in the meanwhile become obsolete the historical analogy to its operating principle continues to shaped the way we use SBMs until today.

Scanning beam microscopy investigation of a specimen is typically comprised of the following steps: 1) scanning the surface for a region of interest (ROI). 2) Recording micrographs of the ROI using detectors specific to the question addressed. 3) If necessary further investigations are performed, e.g. EDX or EBSD analysis. Such an investigation typically takes a few minutes to several hours and results in a couple to several hundred micrographs. Fig. 1 shows a schematic of this approach and the resulting micrographs.

In our contribution we argue that this concept is not only outdated but significantly reduces the usefulness of SBMs: organizing, screening, and interpreting - let alone displaying - the information contained in hundreds of micrographs is challenging at least. Instead we propose a paradigm shift away from understanding the SBM as a tool for creating micrographs towards seeing it as the complex measurement system it is: in essence a device that records detector signals for a certain duration in time at a defined position with high spatial resolution.

In the focus of the new approach is consequentially not the micrograph but the specimen itself. The method aims at creating a virtual representation of the specimen by mapping the recorded signals to a model. This model is created and continually refined during the investigation process. Thereby, unlike in conventional imaging, the signals of all available detectors are recorded and processed simultaneously. Fig. 2 shows an illustration of this process using data from the same series of images as displayed in Fig. 1. Particular micrographs of the ROIs could now be taken from the model but are only one form of representing the acquired data. The modeling itself is not limited to imaging. It is for example possible to use the same model to show dose exposure maps or display elemental composition. Even better, it can be extended to incorporate information in three dimensions and time, e.g. for documenting destructive focused ion beam investigations.

By accepting this paradigm shift away from seeing SBM as micro-photography tools we also open up surprising new possibilities. One example: conventional scanning strategies are designed to create a rectangular image. Typically the region of interest is in the middle of the field of view. Thus, when acquiring high resolution images a large part of the beam time is wasted on imaging uninteresting corner regions. This is not only a efficiency issue but causes severe imaging problems like drift, charging, and contamination. By leaving behind the old approach we would be free to chose the scanning strategy that suits best our particular imaging requirements. 
The shift of focus from pictures to data is not unprecedented in other disciplines. In geography the concept of geographic information systems has been transformative [2]. In our opinion, there is a good chance that "nano-geography" will be equally revolutionizing.

References

[1] M. von Ardenne, Improvements in electron microscopes. Patent GB511204(A) (1939)

[2] M.F. Goodchild, Prog. Hum. Geogr. 15 (1991) 194.

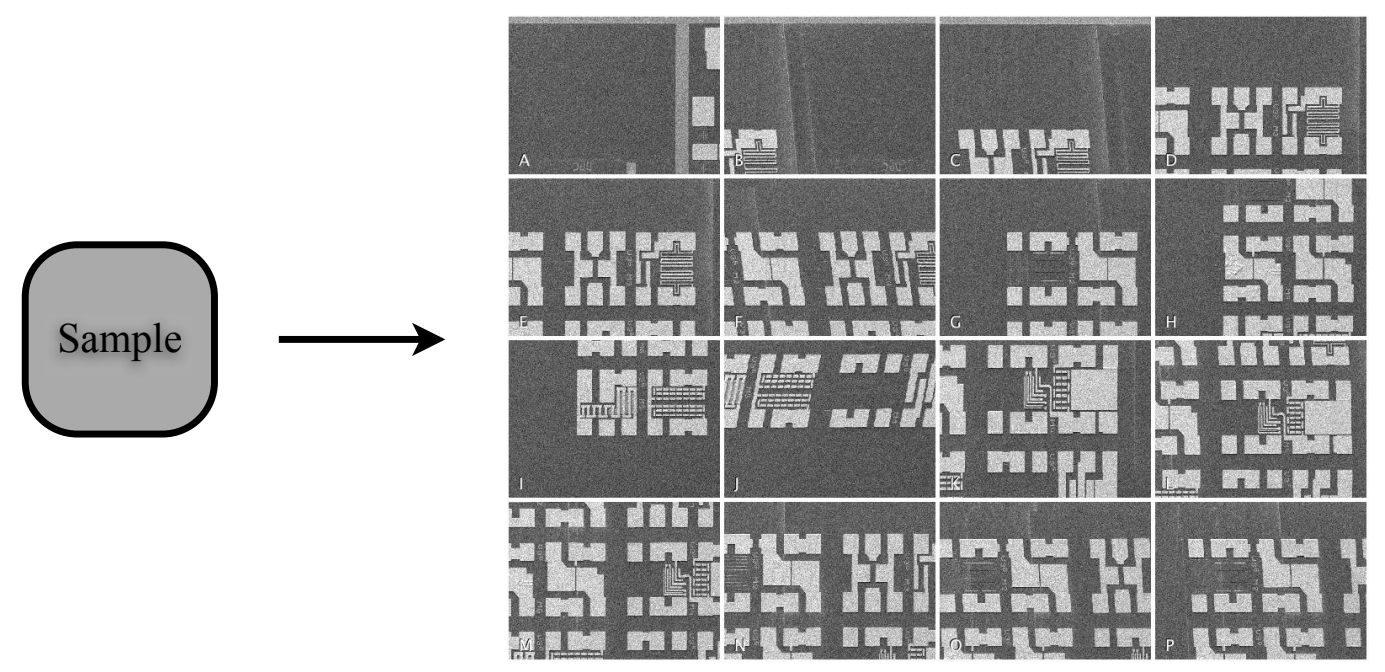

FIG. 1. Convention SEM approach: sample inspection typically results in a small set of micrographs.

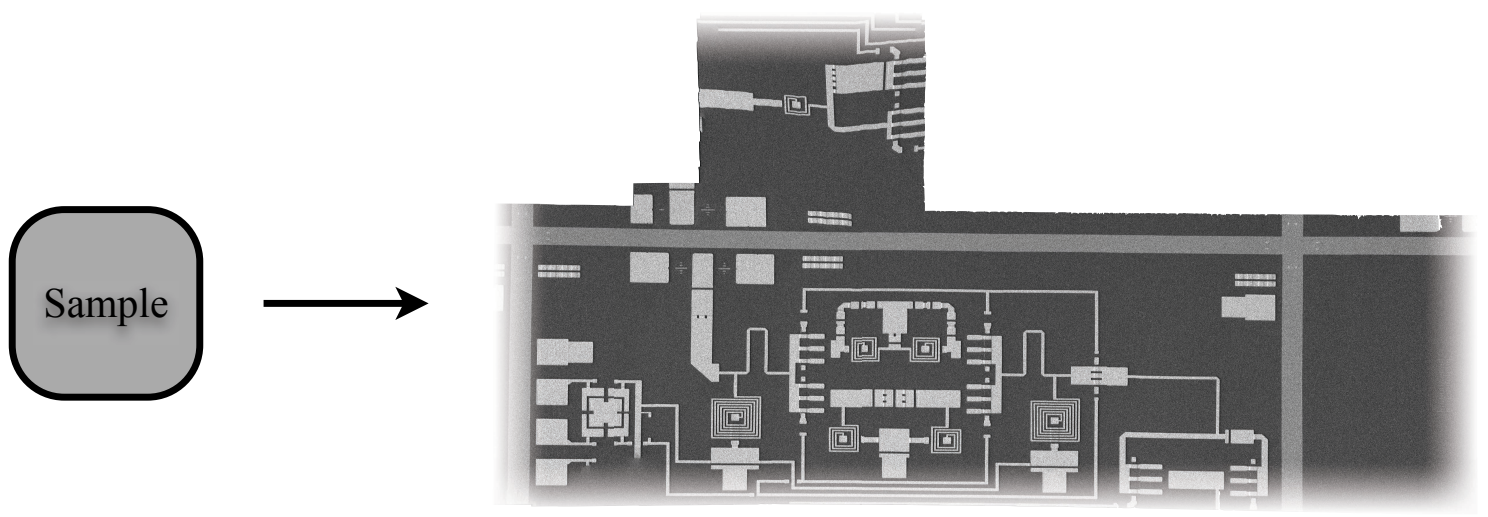

FIG. 2. Nano-geography: reconstruction of the sample surface from a series of 100 micrographs. The reconstruction was done without using stage position information. The images are tilted 90 degree with respect to Fig. 1. 